\title{
When New Technology is an Answer for Old Problems: The Use of Videoconferencing in Cognitive Aging Assessment
}

\author{
Teresa Costa Castanho ${ }^{\mathrm{a}, \mathrm{b}, \mathrm{c}}$, Nuno Sousa $^{\mathrm{a}, \mathrm{b}, \mathrm{c}}$ and Nadine Correia Santos ${ }^{\mathrm{a}, \mathrm{b}, \mathrm{c}, *}$ \\ ${ }^{a}$ Life and Health Sciences Research Institute (ICVS), School of Medicine, University of Minho, Braga, Portugal \\ ${ }^{\mathrm{b}}$ ICVS/3B's, PT Government Associate Laboratory, Braga/Guimarães, Portugal \\ ${ }^{\mathrm{c} C l i n i c a l}$ Academic Center-Braga (CCAB), Braga, Portugal
}

Accepted 7 April 2017

\begin{abstract}
People living with cognitive impairment and dementia require regular screening of their symptomatology and needs. Brief cognitive screening is crucial for assessing these conditions. Such screening can give health professionals a snapshot of the patient's cognitive abilities and help in their monitoring and adaptation. An appropriate administration of brief cognitive screening using telemedicine technology, such as videoconference, can improve access to care and treatment planning. Moreover, the burden that rural and underserved communities often suffer because of limited access to specialty mental health services is also of concern. Herein, in this review, we describe the existing and relevant research regarding the administration of neuropsychological instruments via videoconferencing, and suggest methodological improvements for further studies. To date, only a handful of scientific studies have been published documenting the feasibility and acceptability of videoconferencing among older people and health professionals. Varying in their assessment tools, the studies demonstrate the reliability of cognitive testing and clinical diagnosis of dementia via videoconferencing, as noted by the associations between results of several cognitive tests using it as compared to face-to-face interviews. Further research is required to replicate findings and bring new solutions for their adaptation to a wide range of individuals, health professionals, areas of practice and settings.
\end{abstract}

Keywords: Cognitive testing, dementia, neuropsychology, telemedicine

\section{INTRODUCTION}

Over the last two centuries, the human species worldwide has experienced an impressive progress in health and life expectancy. In fact, currently no country in the world has a lower life expectancy compared to those with the highest life expectancy in 1800, and since 1900 the global average life expectancy

\footnotetext{
*Correspondence to: Nadine Correia Santos, Life and Health Sciences Research Institute, School of Medicine, University of Minho, Campus de Gualtar, 4710-057 Braga, Portugal. Tel.: +351 253 604854; E-mail: nsantos@med.uminho.pt.
}

has more than doubled. However, one of the societal and clinical challenges associated with an aged population is the increased incidence of cognitive impairment, imposing the need for convenient, valid and reliable methods to monitor cognitive functions in the older population. This is a must in order to aid the detection, prevention and/or early intervention, of cognitive decline and/or cognitive (impairment) disorders [1].

In older individuals, standardized neuropsychological evaluations are generally used to screen, characterize and quantify cognitive decline. It is the 
prime criterion of diagnosis, treatment and follow-up of patients with or at-risk for dementia [2]. However, despite of its potential significant benefits, neuropsychological testing has been significantly limited by its need for in-person/face-to-face (FTF) contact and by the costs with travel, overhead, visits and missed visits [2]. For instance, patients who live in rural communities, and/or in remote distances, from medical centers and geriatric services, have difficulties to be screened or evaluated for cognitive performance/status [3, 4]. Also, it has been described that since that transportation is frequently a problem for individuals with dementia, those that are dependent on caregivers may be deprived of this resource [5]. To overcome such limitation(s) there has been a call for increased use of information and communication technologies to render remote cognitive assessment more readily available [6,7], including the use of instruments that allow users to have visual stimuli, rather than simply auditory stimuli (e.g. via telephone assessment), during communication [8, 9]. Using modern information and communication technology, telemedicine blends the needs of patients and technological progress. On this, telemedicine refers to the use of telecommunication technology to deliver health-related services from distance and is thought to be a potential solution in bringing those services to remote and/or underserved areas [10]. Its successful implementation depends on a number of factors, with appropriate use of technology being a necessary basis [11]. Regardless the purpose, there are two main methods in which telemedicine can be conducted: real-time and store-and-forward. In the latter, the information is captured and stored, and then transmitted to the recipient for subsequent reply, it doesn't involve live interaction [12]. Real-time telemedicine allows participants to send and receive information almost immediately. Videoconferencing (VC) is an example.

In this background, besides the use of the telephone, videoconferencing [13] is thought to potentially complement and/or offer potential advantages over FTF assessment [14]. This method of assessment has grown rapidly in recent times partly due to the increasing availability and affordability of computers with video capacity and of communication by internet. Videoconferencing has been the most common application of telemedicine care and has been used to carry out cognitive assessments, to conduct psychiatric interviews and consultations, and to help in management of conditions such as stroke, diabetes mellitus, chronic illness, cancer, depression and post-traumatic stress [15]. Besides improving the assistance to (older) individuals who already have (mild) cognitive impairment, it can also reach those that still without deficit and/or professionally active, those living in rural settings and with insufficient healthcare resources, avoiding time and money loss due to travel and time missed at work. By using videoconferencing technology, health professionals and patients can reduce the costs with regular clinic visits. Besides that, videoconference also allows hospitals to create networks to provide each other with support and improve care. By easily sharing their knowledge, health professionals can provide faster and more efficient services through earlier diagnosis and better management of, for instance, chronic disease [10]. At last, videoconferencing may as well improve the capacity to reach a great number of individuals located at multiple stations in a short period of time, offering timely responses and aiding in epidemiological data collection. In this line, Dorsey and colleagues evaluated in a randomized clinical trial the feasibility, effectiveness and economic benefits of videoconference care for 20 individuals with Parkinson disease in their home [16]. They reported that videoconference offered similar clinical control, and saved participants 100 miles of traveling and $3 \mathrm{~h}$ of time.

Herein we describe the existing and relevant studies regarding the administration of neuropsychological tests via VC, and suggest methodological improvements for future research.

\section{METHODS}

The databases Pubmed and Psychinfo were searched for relevant journal articles published in English, using a combination of the search terms with reference to applicability of cognitive tests via VC: telemedicine, videoconferencing, aging (ageing), cognition, Alzheimer, dementia and neuropsychological assessment. Though there are different ways in which telemedicine applications are being used to provide neuropsychological services, this manuscript was limited only to the discussion of the use of videoconferencing. On this, studies should address a videoconference approach between a health professional and an older patient (aged above 50 years old) for the purpose of diagnosis and/or cognitive assessment, with relevant reporting of statistical results. Publications were limited for the period of 2000 to 2016. Titles and abstracts were evaluated as a first 
step and then full-text articles were read for their relevance to this review. In addition, bibliographies from the obtained articles were screened for relevant publications. The search was conducted between May 2016 and January 2017, with a total final of 20 studies with the inclusion characteristics properly identified.

\section{RESULTS}

\section{Accuracy of cognitive testing in cognitive impairment using videoconferencing}

In cognitive impairment care, telemedicine has been used in the areas of patient diagnosis/ assessment. The evaluation of cognitive functioning via $\mathrm{VC}$ using standardized assessment tools has been shown to correlate well with FTF testing (e.g., $[2,3,17-20])$. Some of cognitive instruments frequently used via VC include the Mini-Mental State Examination (MMSE), Standardized MMSE, Clock Drawing test, Short Blessed test, Rowland Universal Dementia Assessment Scale (RUDAS), Digit Span, Hopkins Verbal Learning test - Revised (HVLT-R) and tests of verbal fluency. The accuracy of a range of cognitive tests that are commonly used in dementia evaluations was tested by Cullum et al. in 14 patients with mild cognitive impairment and 19 patients with mild to moderate Alzheimer's disease [2]. The study demonstrated the comparability of VC-based and traditional in-person testing conditions, indicating robust correlations between the two modalities [e.g., MMSE $(r=0.89)$, Boston Naming test $(r=0.88)$, digit span $(r=0.81)$, category fluency $(r=0.58)$, and letter fluency $(r=0.83)$ ], providing a good indicator that cognitive assessment via $\mathrm{VC}$ may to be a valid and reliable means to conduct neuropsychological evaluations in older individuals with cognitive impairment. A few years later, Cullum and colleagues [21] increased the sample size of their investigation to 202 individuals (83 with cognitive impairment and 119 healthy controls) from both rural and urban settings. Using the approach previously described, they found highly similar results across the VCbased remote and in-person assessment conditions, with significant associations between test scores. Although they had used a larger sample, the authors suggest that the battery of tests applied should have been comprehensive in order to verify which neuropsychological tests can be administered validly by $\mathrm{VC}$, and those that are not so suitable. In a study that focused on the effectiveness of language evaluation in mild Alzheimer's disease patients, Vestal et al. compared VC with in-person language assessments [5]. Findings from the Wilcoxon signed rank test revealed no significant differences in performance on each of the 5 language tests between $\mathrm{VC}$ and in-person modalities, and, altogether, $\mathrm{VC}$ proved to be promising for speech and language evaluation services in dementia [5].

Other studies have focused on specific cognitive tests, such as the Mini-Mental State Examination (MMSE; [17, 22, 23]). Applying the MMSE via telemedicine, Loh et al. aimed to determine the inter-rater reliability of this instrument through VC, as compared to FTF administration, in 20 geriatric patients with diversified diagnoses (normal, dementia and depression) $[18,23]$. Data indicated that assessments performed by distance with the MMSE using VC yielded similar results to direct evaluations. Timpano et al., whose study validated an Italian version of the 28-item VC-based MMSE (VMMSE) in cognitively impaired and healthy subjects, also concluded that $\mathrm{VC}$ is a valid method for clinical and research screening [24]. The results demonstrated that VMMSE has high levels of sensitivity and specificity for the optimal cutoff identification and an accuracy of 0.96 . Moreover, VC was preferred over FTF, as it allowed real time communication between the health professional and the patient. Ciemins and colleagues examined the reliability of MMSE administration via remote way in a sample of 72 patients with diabetes [22]. The main focus was to assess the auditory and visual components of the administration. Findings revealed that $80 \%$ of individual items demonstrated remote to FTF agreement of $\geq 95 \%$ and all item were $\geq 85.5 \%$. However, due to the study design only the differences between the scores of the two methods were documented.

In more recent studies, other tests have also been evaluated for their feasibility and reliability when comparing VC with FTF test conditions. GalushaGlasscock and colleagues [25] found that the Repeatable Battery for the Assessment of Neuropsychological Status (RBANS) could be administered via VC, with similar scores obtained when the RBANS was administered in-person. Using three different assessment approaches (VC, telephone and in-person), Castanho et al. [20] sought to determine if the use of $\mathrm{VC}$ with the Telephone Interview for Cognitive Status Modified - Portuguese (TICSMPT; [26]) provided comparable information to an in-clinic evaluation. Findings indicated that the VCbased testing yielded similar results to the traditional 
FTF administration and that cognitive test assessment via $\mathrm{VC}$ is a tool for consideration by the health professionals to reliably follow-up their patients who live in different settings.

\section{Dementia diagnostic services in clinical settings using videoconferencing}

Barton et al. described a VC neuropsychological assessment in a clinical setting [19]. Using VC, a comprehensive battery of tests comprising clinical and neurologic assessments was performed to 15 veterans. Results indicated that VC is a feasible method to provide consultation and care to people who would otherwise not have access to it. It was also possible to reach a working diagnosis and recommend relevant treatments for each patient. Additionally, with a clinical sample, Harrell and colleagues demonstrated that the majority of patients evaluated by them via telemedicine had an inaccurate diagnosis at the time of the FTF consultation [15]. With the administration of a neuropsychological battery of tests via VC, the investigators identified missed and unrecognized mental health treatments needs in over $77 \%$ of the patients referred. Telemedicine, thus, proved to be a valuable resource to clarify cognitive and psychiatric diagnoses. Moreover, a study by Martin-Khan and colleagues designed to validate the diagnosis of dementia via VC using a sample of 205 patients demonstrated a significant agreement for the primary outcome "Does the patient have dementia?" between VC and in-person testing conditions [27].

\section{Users' and cultural acceptability of videoconferencing}

Research on VC-based neuropsychological assessment has been promising in terms of subject satisfaction and acceptability. Parikh et al. indicate that when asked about their preference, $98 \%$ of the individuals evaluated in the traditional FTF condition and via VC expressed high satisfaction with both test modalities, and $60 \%$ indicated no preference for a test condition [28]. After completing a questionnaire that evaluated the usefulness of the VC interaction, its efficacy compared to FTF or telephone testing modalities and willingness to try VC again, the same high satisfaction level was seen in the study of Shores et al. with a group of veterans [3]. In addition to the good acceptability, there was $100 \%$ agreement in dementia diagnoses between in-person and VC assessments. In another study, Grosch and colleagues documented that the individuals did not experience any difficulties in adapting to the testing environment or procedures, and they expressed satisfaction with the VC assessment [29]. In turn, with a sample of healthy volunteers, the study of Hildebrand and colleagues revealed a preference for in-person testing (44\%), but because $39 \%$ of the individuals indicated no preference the authors to conclude good acceptance of VC-based assessments [14].

Telemedicine diagnosis and assessment have also been welcomed in Spanish-speaking and Native American populations from remote and rural settings where access to specialized care is restricted [13, $30,31]$. Using a Spanish-language battery of cognitive tests via VC, Vahia and colleagues compared it to in-person and found no significant differences between cognitive scores [30]. Additionally, the study of Weiner et al., in a sample of individuals with dementia of the Choctaw Nation, suggested that the use of $\mathrm{VC}$ to diagnose and treat cognitive disorders was a feasible alternative to the traditional FTF examination [13]. High levels of acceptability of VC use were found amongst this specific sample, with only $3 \%$ not showing for $\mathrm{VC}$ meetings and 2 individuals refusing to be examined afterwards [13]. Wadsworth and colleagues [31] also adds to the growing literature on the feasibility and reliability for remote neuropsychological assessment by demonstrating that test scores were highly similar between VC and face- to-face conditions in their sample of American Indians.

\section{DISCUSSION}

The state of the art described here provides evidence that VC is feasible and acceptable among many older people and health professionals. Studies have demonstrated the reliability of cognitive testing via video-telemedicine, as noted by the correlations between results of various cognitive tests using VC as compared to in-person interview. Specifically, MMSE via telemedicine (e.g., $[18,22,23]$ ) can be as accurate as FTF, as well as particularly useful in documenting cognitive stability or decline. Other studies have compared the results of several neuropsychological tests, through FTF and VC, in patients cognitively intact and with dementia. These studies did not find any significant difference between the two testing modalities and showed that patient acceptance and satisfaction with telemedicine is high. Moreover, comparisons of diagnosis made FTF and via 
VC suggested that clinical diagnosis of dementia via telemedicine consultation is valid and reliable [3, 23].

Although VC increases access to particular services, there are inherent challenges specific to its use. Concerning the administration of neuropsychological tests, the ones that are the standard of care for assessment of older adults include measures that are sometimes not feasible for VC administration [15]. One should be careful in that videoconferencing interaction must be as similar as possible to the traditional in-person administrations as much as possible. If test instructions are modified significantly for VC administration, standardized scoring protocols, and perhaps different norms, may be needed [10]. Health professionals have an ethical responsibility to maintain psychometric standards and test integrity. Also, participants tested by the same health professional in both conditions (VC and FTF) may inflate test-retest correlations. Furthermore, using and learning new technology often demands keen perceptual and cognitive abilities which tend to decline as we get older. Therefore, the use of $\mathrm{VC}$ can be limiting for patients with significant sensory and auditory problems or those with more severe confusion or communication difficulties [32]. Finally, it should be kept in mind that results cannot yet be extrapolated or generalized because, to date, most studies have been conducted in small and non-randomized samples, and in specific settings.

Related to the technology itself, ensuring that data and equipment are secure is crucial. This includes the use of a VC system with appropriate levels of data encryption to anonymize the messages between the patient and health professional, electronic signatures and privacy safeguards, so to prevent data loss and maintain confidentiality. However, since that health professionals have a limited control when patient information is transmitted wirelessly, these potential risks should be explicit in the informed consent $[10,33]$. Technical problems concerning technology can also compromise the effectiveness of assessments via $\mathrm{VC}$. If there is poor quality of transmission, relevant testing information may be lost and a clear view of patients' behavior may be disrupted. If there are lapses in sound, camera resolution or internet band width, the person that is administering the test has to repeat the instructions and, thus, the validity of some results, their scoring and interpretation may be compromised [10]. However, it must be pointed out that recent technological advancements have been phenomenal in mitigating many of the shortcomings found in older studies.
Early studies suggest that VC technology is a particularly useful medium to help extend neuropsychological assessments specifically to rural elderly communities [3, 14] However, they may face some issues with the necessary broadband infrastructure of telemedicine. In fact, remote assessments amount to nothing if there isn't the network infrastructure to support it. Related to this, another challenge that can be found is to ensure that health professionals have the necessary information/education and technical competence to manage and adapt to any new health services they want to deliver [33].

Taking into account VC limitations, some recommendations are necessary for its use. First, before incorporating an instrument into a $\mathrm{VC}$ assessment, its reliability in the administration needs to be confirmed in the population setting attending to factors such as education level, cultural aspects and exposure to technology. Those who administer neuropsychological testing should also guarantee competence in the practice of cognitive assessment via telemedicine, including appropriate educational background, and proper training [22]. It should also be carried out an evaluation of the possible effects of sensory impairment and presence of psychiatric disorders on performance and subject's reactions to $\mathrm{VC}$, previous to the assessment itself. A critical factor in acceptance of technology is its ease of use. Telemedicine through video should be simple and appropriate for the individual's competence. This is especially pertinent for people with dementia who usually have reduced processing speed, less manual dexterity and low visual acuity.

Remote assessments may inhibit key components of communication that are important for the patienthealth professional relationship. For instance, due to the need to look into a camera when videoconferencing, the individual often makes contact with the other's image on the screen and not to the camera. This phenomenon may hinder the establishment and maintenance of eye contact, an aspect that has been associated with rapport and empathy bond. In order to ensure care and due considerations, it is suggested an initial interaction via VC for several minutes to help in developing rapport and familiarize the patient with the VC environment and equipment [10]. An important concern is also what to do if a patient becomes anxious or has a medical emergency during a remote assessment session. Having a security plan can be a solution. This should include procedures such as emergency contacts from where the patient resides [34]. Engaging someone who can intervene locally 
in technical problems, such as a family member or a close friend, is crucial.

Additionally to the need of high-quality videoconferencing equipment to protect against breaches of privacy and confidentiality, health professionals should ensure no other subjects enter the room at any time during the assessment without patient's consent [10]. Although there are few guidelines to orient health professionals as to ethical and utilization of telemedicine, the American Telemedicine Association and the American Psychological Association (APA) made available specific guidelines to highlight best practices and type of recommended conduct when providing psychological services using technology [34, 35]. In 2013, the APA Task Force developed their recommendations based on identified data and experiences and then, they examined the research produced on the effectiveness, ethical concerns, legal questions, and practical concerns. These guidelines are necessary in research and clinical settings in order to ensure the protection of both patient and health professional, and that assessments and other services via VC interactions are similar to the traditional face-to-face approach [10]. At last, future studies should consider the use of larger and mixed diagnostic samples, as well as focus on subjective assessment of satisfaction/acceptability of telemedicine and not only administering cognitive tests. Further research is, thus, still warranted to fully establish the feasibility of conducting remote cognitive assessments and dementia diagnosis in real-life than experimental settings.

\section{Conclusions}

Telemedicine represents an excellent approach in the incorporation of technology, such as videoconferencing, in bridging the distance between health professionals and patients. On this, VC can be a useful tool in the screening and signaling patients with specific needs for further examination or followup. Moreover, the use of 'telemedicine' in cognitive assessment has the potential to support people with dementia while improving patients' quality of life and reduce healthcare costs [36]. The current stateof-the-art indicates for its use being as effective as an in-person evaluation, and an acceptable approach to assess those in underserved and remote areas. Still, despite the promising results, further research is required to replicate findings and bring a new solution to an old problem: to reach all individuals.

\section{ACKNOWLEDGMENTS}

This work was partially funded by the European Commission (FP7): "SwitchBox" (Contract HEALTH-F2-2010-259772), and co-financed by the Portuguese North Regional Operational Program (ON.2 - O Novo Norte), under the National Strategic Reference Framework (QREN), through the European Regional Development Fund (FEDER), and by the Fundação Calouste Gulbenkian (Portugal) (Contract grant number: P-139977; project "Better mental health during ageing based on temporal prediction of individual brain ageing trajectories (TEMPO)"). TCC is recipient of a doctoral fellowship from the Portuguese Foundation for Science and Technology (FCT, Portugal; SFRH/BD/90078/2012) and, NCS of a Research Assistantship by the Portuguese Foundation for Science and Technology (FCT, Portugal) through the "FCT Investigator Programme $(200 \infty$ Ciência)".

\section{CONFLICT OF INTEREST STATEMENT}

The research was conducted in the absence of any commercial or financial relationships that could be construed as a potential conflict of interest.

\section{REFERENCES}

[1] Eyre H, Baune B, Lavretsky H (2015) Clinical advances in geriatric psychiatry: A focus on prevention of mood and cognitive disorders. Psychiatr Clin North Am 38, 495-514.

[2] Cullum CM, Weiner MF, Gehrmann HR, Hynan LS (2006) Feasibility of telecognitive assessment in dementia. Assessment 13, 385-390.

[3] Shores MM, Ryan-Dykes P, Williams RM, Mamerto B, Sadak T, Pascualy M, Felker BL, Zweigle M, Nichol P, Peskind ER (2004) Identifying undiagnosed dementia in residential care veterans: Comparing telemedicine to inperson clinical examination. Int J Geriatr Psychiatry 19, 101-108.

[4] Morgan DG, Crossley M, Kirk A, D'Arcy C, Stewart N, Biem J, Forbes D, Harder S, Basran J, Dal Bello-Haas V, McBain L (2009) Improving access to dementia care: Development and evaluation of a rural and remote memory clinic. Aging Ment Health 13, 17-30.

[5] Vestal L, Smith-Olinde L, Hicks G, Hutton T, Hart J Jr (2006) Efficacy of language assessment in Alzheimer's disease: Comparing in-person examination and telemedicine. Clin Interv Aging 1, 467-471.

[6] Tyrrell J, Couturier P, Montani C, Franco A (2001) Teleconsultation in psychology: The use of videolinks for interviewing and assessing elderly patients. Age Ageing 30, 191-195.

[7] Lee JH, Kim JH, Jhoo JH, Lee KU, Kim KW, Lee DY, Woo JI (2000) A telemedicine system as a care modality for 
dementia patients in Korea. Alzheimer Dis Assoc Disord 14, 94-101.

[8] Jastrzembski TS, Roring RW, Charness NH (2006) Videoconferencing technology as environmental support for older adults. Proc Hum Factors Ergon Soc Аnпи Meet 50, 175179.

[9] Martin-Khan M, Wootton R, Whited J, Gray LC (2011) A systematic review of studies concerning observer agreement during medical specialist diagnosis using videoconferencing. J Telemed Telecare 17, 350-357.

[10] Grosch MC, Gottlieb MC, Cullum CM (2011) Initial practice recommendations for teleneuropsychology. Clin Neuropsychol 25, 1119-1133.

[11] Fatehi F, Armfield NR, Dimitrijevic M, Gray LC (2015) Technical aspects of clinical videoconferencing: A large scale review of the literature. J Telemed Telecare 21, 160166.

[12] Raad MW, Sheltami T, Shakshuki E (2015) Ubiquitous tele-health system for elderly patients with Alzheimer's. Procedia Comput Sci 52, 685-689.

[13] Weiner MF, Rossetti HC, Harrah K (2011) Videoconference diagnosis and management of Choctaw Indian dementia patients. Alzheimers Dement 7, 562-566.

[14] Hildebrand R, Chow H, Williams C, Nelson M, Wass P (2004) Feasibility of neuropsychological testing of older adults via videoconference: Implications for assessing the capacity for independent living. J Telemed Telecare 10, 130134.

[15] Harrell KM, Wilkins SS, Connor MK, Chodosh J (2014) Telemedicine and the evaluation of cognitive impairment: The additive value of neuropsychological assessment. J Am Med Dir Assoc 15, 600-606.

[16] Dorsey ER, Venkataraman V, Grana MJ, Bull MT, George BP, Boyd CM, Beck CA, Rajan B, Seidmann A, Biglan KM (2013) Randomized controlled clinical trial of "virtual house calls" for Parkinson disease. JAMA Neurol 70, 565570.

[17] Saligari J, Flicker L, Loh PK, Maher S, Ramesh P, Goldswain P (2002) The clinical achievements of a geriatric telehealth project in its first year. $J$ Telemed Telecare 8, 53-55.

[18] Loh PK, Ramesh P, Maher S, Saligari J, Flicker L, Goldswain P (2004) Can patients with dementia be assessed at a distance? The use of Telehealth and standardised assessments. Intern Med J 34, 239-242.

[19] Barton C, Morris R, Rothlind J, Yaffe K (2011) Videotelemedicine in a memory disorders clinic: Evaluation and management of rural elders with cognitive impairment. Telemed J E Health 17, 789-793.

[20] Castanho TC, Amorim L, Moreira PS, Mariz J, Palha JA, Sousa N, Santos NC (2016) Assessing cognitive function in older adults using a videoconference approach. EBioMedicine 11, 278-284.

[21] Munro Cullum C, Hynan LS, Grosch M, Parikh M, Weiner MF (2014) Teleneuropsychology: Evidence for video teleconference-based neuropsychological assessment. J Int Neuropsychol Soc 20, 1028-1033.

[22] Ciemins EL, Holloway B, Coon PJ, McClosky-Armstrong T, Min SJ (2009) Telemedicine and the mini-mental state examination: Assessment from a distance. Telemed $J E$ Health 15, 476-478.

[23] Loh P-K, Donaldson M, Flicker L, Maher S, Goldswain P (2007) Development of a telemedicine protocol for the diagnosis of Alzheimer's disease. J Telemed Telecare 13, 90-94.

[24] Timpano F, Pirrotta F, Bonanno L, Marino S, Marra A, Bramanti P, Lanzafame P (2013) Videoconference-based mini mental state examination: A validation study. Telemed $J E$ Health 19, 931-937.

[25] Galusha-Glasscock JM, Horton DK, Weiner MF, Cullum CM (2016) Video teleconference administration of the repeatable battery for the assessment of neuropsychological status. Arch Clin Neuropsychol 31, 8-11.

[26] Castanho TC, Portugal-Nunes C, Moreira PS, Amorim L, Palha JA, Sousa N, Correia Santos N (2016) Applicability of the Telephone Interview for Cognitive Status (Modified) in a community sample with low education level: Association with an extensive neuropsychological battery. Int J Geriatr Psychiatry 31, 128-136.

[27] Martin-Khan M, Flicker L, Wootton R, Loh PK, Edwards H, Varghese P, Byrne GJ, Klein K, Gray LC (2012) The diagnostic accuracy of telegeriatrics for the diagnosis of dementia via video conferencing. J Am Med Dir Assoc 13, 8.

[28] Parikh M, Grosch MC, Graham LL, Hynan LS, Weiner M, Shore JH, Cullum CM (2013) Consumer acceptability of brief videoconference-based neuropsychological assessment in older individuals with and without cognitive impairment. Clin Neuropsychol 27, 808-817.

[29] Grosch MC, Weiner MF, Hynan LS, Shore J, Cullum CM (2015) Video teleconference-based neurocognitive screening in geropsychiatry. Psychiatry Res 225, 734-735.

[30] Vahia IV, Ng B, Camacho A, Cardenas V, Cherner M, Depp CA, Palmer BW, Jeste DV, Agha Z (2015) Telepsychiatry for neurocognitive testing in older rural Latino adults. Am J Geriatr Psychiatry 23, 666-670.

[31] Wadsworth HE, Galusha-Glasscock JM, Womack KB, Quiceno M, Weiner MF, Hynan LS, Shore J, Cullum CM (2016) Remote neuropsychological assessment in rural American Indians with and without cognitive impairment. Arch Clin Neuropsychol 31, 420-425.

[32] McEachern W, Kirk A, Morgan DG, Crossley M, Henry C (2008) Reliability of the MMSE administered in-person and by telehealth. Can J Neurol Sci 35, 643-646.

[33] Adjorlolo S (2015) Can teleneuropsychology help meet the neuropsychological needs of Western Africans? The case of Ghana. Appl Neuropsychol Adult 22, 388-398.

[34] Joint Task Force for the Development of Telepsychology Guidelines for Psychologists (2013) Guidelines for the practice of telepsychology. Am Psychol 68, 791-800.

[35] Krupinski EA, Antoniotti N, Bernard J (2013) Utilization of the American Telemedicine Association's clinical practice guidelines. Telemed J E Health 19, 846-851.

[36] Bossen AL, Kim H, Williams KN, Steinhoff AE, Strieker M (2015) Emerging roles for telemedicine and smart technologies in dementia care. Smart Homecare Technol Telehealth 3, 49-57. 